\title{
High-fidelity Aerostructural Optimization of a High Aspect Ratio Tow-steered Composite Wing
}

\author{
Timothy R. Brooks, * Joaquim R. R. A. Martins ${ }^{\dagger}$ \\ University of Michigan, Ann Arbor, Michigan, United States \\ Graeme J. Kennedy, \\ Georgia Institute of Technology, Atlanta, Georgia, United States
}

\begin{abstract}
In the last 30 years since their introduction into aerospace applications, composites have become increasingly used, making up as much as $50 \%$ of modern aircraft by weight. Considering this fact, it is surprising that most aircraft today are only scratching the surface of the true potential of composite technology with traditional uniaxial fibers. With the introduction of Automated Fiber Placement machines (AFP), the tow direction in laminae is now allowed to be steered spatially throughout each layer. This process is known as composite tow steering and has been shown to have improved performance over its uniaxial fiber counterpart with no additional weight penalty. With modern aircraft wings moving toward higher aspect ratios, which inevitably leads to larger deflections, it is reasonable to assume that a tow-steered composite structure can be tailored to outperform its unsteered counterpart. However, given the highly coupled nature of the aerodynamics and structural response of the problem it is not obvious nor intuitive to find the composite fiber pattern that would yield an optimum result. To address this issue, we develop a framework for the simultaneous design of aerodynamic shape, structural sizing, and tow steering angles, while considering the wing flexibility. The aerodynamics are modeled using RANS CFD, while the structure is analyzed using a detailed finite-element model. Using this framework we perform two fuel burn optimization problems are performed for a high aspect ratio $(A R=13.5)$ baseline wing: one using a tow-steered structure and another with no steering, unsteered. The optimization was performed with respect to tow-steering design variables, wing airfoil shapes and twist, as well as structural thicknesses. Along with including design variables which controlled the tow-steering parameterization, geometric shape variables were also included to vary the shape of the wing. The tow-steered wing was able to decrease the wing mass by $13 \%$ relative to the baseline unsteered wing.
\end{abstract}

\section{Introduction}

With advent of AFP machines designers have now gained a greater degree of freedom over the design of the structure. Using AFP, the local stiffness properties of the structure can be changed to a greater degree, and thus we can tailor the structural deformations under a prescribed load more effectively. When this concept is applied to a wing, it should be possible to design a composite wing that deflects during cruise in such a way that the lift distribution on the wing is closer to elliptical, reducing the induced drag and improving the fuel burn, while shifting the loads inboard at maneuver conditions, reducing the structural weight required to design the wing. This approach is known as passive load alleviation and has been shown to be achievable with both metallic and composite designs through high fidelity aerostructural optimization, $[1,2,3]$. The assumption for a tow-steered wing is that the degree of passive load alleviation can be increased relative to conventional wing designs.

Optimization of composite materials with spatially varying stiffness properties has been a growing topic of interest. Hvejsel et al. [4], Kennedy and Martins [5], and others have considered composite laminates with spatially varying stiffness properties, however the design was set at a number of discrete patches Where the tow orientations for each layer were constrained to a set of discrete tow orientations $\left(0^{\circ}, \pm 45^{\circ}, 90^{\circ}\right.$, etc.). The difference between such a structure and a tow-steered structure is that the orientations defined for the tow-steered structure are allowed to vary

\footnotetext{
*Ph.D. Candidate, Department of Aerospace Engineering, AIAA Member

${ }^{\dagger}$ Professor, Department of Aerospace Engineering, AIAA Associate Fellow

¥Assistant Professor, School of Aerospace Engineering, AIAA Member
} 
continuously and take any value, giving the structure much more design freedom. Studies done by Jutte al.[6] and Stodieck et al.[7] have shown some of the potential benefits that tow-steering optimization has to offer when applied to a composite wing, Jutte al.[6] were able to show up to $15 \%$ in wing weight reduction and $47 \%$ in aggregate stress reduction when compared to a traditional composite layup. These studies offered impressive results but were constrained to a panel code for the aerodynamics and Genetic Algorithms (GA), inherently limiting dimension of the design space to $\mathcal{O}(10)$ design variables. The purpose of this paper is to perform a high fidelity single point aerostructural optimization of a 13.5 aspect ratio tow steered composite wing using gradient based optimization methods. Using this optimization we quantify the benefits of a tow steered composite wing box when compared to an unsteered composite wing box.

\section{Method}

In order to quantify the benefits of passive load alleviation, it is necessary to consider the coupling between aerodynamics and structural deformation that is particularly strong in high aspect ratio wings. To this end we perform a static aeroelastic analysis, where the internal structural forces are in equilibrium with the aerodynamic loads. The analysis features a RANS based Computational Fluid Dynamics (CFD) solver to compute aerodynamic loads, coupled to a Computational Structural Mechanics (CSM) solver, to compute structural deformations. The optimization and analysis scheme used to solve this problem is implemented in the MACH (MDO for Aircraft Configurations with High fidelity) framework [2], described throughout the rest of this section. This framework has been well tested, being used in a number of other aerostructural optimization studies $[1,8]$.

\section{A. Shape parametrization}

The parameterization of the shape of the wing for optimization was performed through the use of a Free Form Deformation (FFD) algorithm [9]. The implementation of this algorithm is described by Kenway and Martins [1], in which the wing is embedded in a volume with control points distributed on the surface. When these control points are perturbed, the embedded geometry of the wing is likewise deformed. This gives the optimizer full control of the geometry of the wing. In addition control over the cross-sectional shape of the wing these control points allow for the geometrical twist of the wing to be varied at span-wise locations. In addition to the wing FFD, a smaller FFD volume is also applied to the horizontal stabilizer of the model. This sub-FFD gives the optimizer control over the incidence of the stabilizer, allowing the optimizer to trim the pitch of the aircraft for each flight condition.

\section{B. Mesh Movement}

To update the CFD volume mesh with the deformations provided by the CSM solution, a mesh warping algorithm is required. This work uses an inverse distance weighting method, such as that described by Uyttersprot [10]. This algorithm first takes the displacements and rotations computed on the surface of the wing provided by the structural solution, and then extrapolates these deformations throughout the volume of the mesh toward the far-field. The benefit of this method is that the mesh quality near the surface of the wing is maintained throughout the optimization, and the accuracy and convergence properties of the CFD are likely maintained.

\section{Aerodynamic Solver}

In order to accurately predict aeroelastic benefits, a high-fidelity aerodynamic solver is required. To this end, a second order finite-volume CFD solver, SUMad (formerly SUmb) [11], is used. SUMad is capable of solving the steady Reynolds-Averaged Navier-Stokes (RANS) equations for structured block meshes using a single equation SpalartAllmaras (SA) turbulence model. The solver computes aerodynamic functions of interest such as lift and drag, as well as the corresponding sensitivities of these functions using an efficient adjoint method implemented through Automatic Differentiation (AD) and developed by Mader et al. [12] and Lyu et al. [13].

\section{Structural Solver}

To solve the problems considered in this paper, a high-fidelity structural solver is also required. Structures seen in airframes feature numerous thin shells that lead to large condition numbers when assembling the linear system for 
the finite-element problem, leading to a loss of accuracy. The MACH framework utilizes the Toolkit for Analysis of Composite Structures (TACS) [14] as its CSM solver. TACS utilizes a parallel direct factorization method to accurately and efficiently solve these inherently poorly conditioned problems. TACS is capable of computing both structural functions of interest such as buckling and failure values as well as their corresponding sensitivities with respect to large numbers of design variables at low computational cost using an efficient adjoint method.

\section{E. Optimizer}

For this work we decided to use a gradient based optimization method. The reason for this is because the problems considered feature a large number, $\mathcal{O}(100)$, of design variables. It is for this reason that the solvers described above are capable of computing sensitivities with respect to large numbers of design variables for relatively low cost, using adjoint methods, and a coupled-adjoint method was implemented in MACH [2]. All optimization problems in this work are performed using SNOPT (Sparse Nonlinear OPTimizer) [15] a gradient-based quasi-Newton optimizer designed to perform well for optimization problems featuring many sparse nonlinear constraints. The optimization tool is then wrapped by a Python interface using pyOPT [16], a Python module with a suite of tools for nonlinear optimization problems.

\section{Laminate Parameterization}

For the wing design optimization, only the upper and lower skins of the wing box are considered to be tow steered. In order to model the effects of tow steering it is necessary to allow the laminate variables to parametrically vary throughout the skin of the wing. Rather than optimizing each layer individually, leading to an unwieldy number of design variables, the laminate of each skin is defined by four unique tow steered patterns, defining the local tow orientation for each layer, and a laminate thickness distribution, $t(u, v)$, where $u$ and $v$ are the parametric coordinates defining the surface. During the tow steering procedure each pattern is assumed to be repeated through the thickness of the laminate at a frequency corresponding to that pattern's ply fraction. The laminate is assumed to be thick enough and therefore composed of many repeated layers. This assumption has two benefits. The first is that since the laminate thickness is much larger than the ply thickness, the optimization problem can be treated as continuous with respect to the laminate thickness. Thus, the discrete nature of the plies is neglected. The second benefit is that because the laminate consists of a few unique layers repeated many times through the thickness of the laminate, we can define a homogenized material stiffness based on the relative frequency of occurrence through the thickness-the ply fraction - of each unique layer so long as the stacking pattern does not vary through the thickness. This allows us to safely neglect stacking sequence in the optimization for sufficiently thick laminates. This procedure is explained further in the following section.

In our study, the first pattern is defined as the main tow path layer and makes up $62.5 \%$ of the through thickness composition of the laminate at any point. The three remaining patterns make up $12.5 \%$ each of the through thickness composition and are defined as offsets of $45^{\circ},-45^{\circ}$, and $90^{\circ}$ relative to the main steer pattern at every point, such that in the laminate the plies are balanced with respect to the main steered pattern, also referred to as the $0^{\circ}$ pattern, at every point. This means that the laminate properties on the tow steered surface are completely defined by the distribution of the laminate thickness, $t(u, v)$, and the main steering fiber orientation, $\theta_{0}(u, v)$. In order to parameterize these two distributions B-spline control points are distributed throughout the surface of the wing box. The design variables specified by the optimizer, $t^{c p}$ and $\theta^{c p}$, are set at these control points, which then define the laminate parametric properties. An example of the process used to define the main staring orientation and subsequently the four unique tow steered patterns is illustrated in Figure 1. The laminate properties are then interpolated to find their values at the nodes of the finite element model using B-spline interpolation. The B-splines reduce the dimensionality of the design space from $\mathcal{O}\left(10^{4}\right)$ to $\mathcal{O}\left(10^{2}\right)$.

\section{A. Tow-steering Constraints}

In this work we consider three constraints to ensure that the tow in the laminate varies smoothly and is thus is realizable with an AFP machine. The first of these constraints is on the minimum turning radius of the tow path, $r_{\min }$. This constraint is defined by the manufacturer to prevent the pre-preg tape from twisting over itself as the tow is laid down by the head of the AFP machine. To compute this value, the tow curvature, $\kappa(u, v)$, must first be defined. This can be 


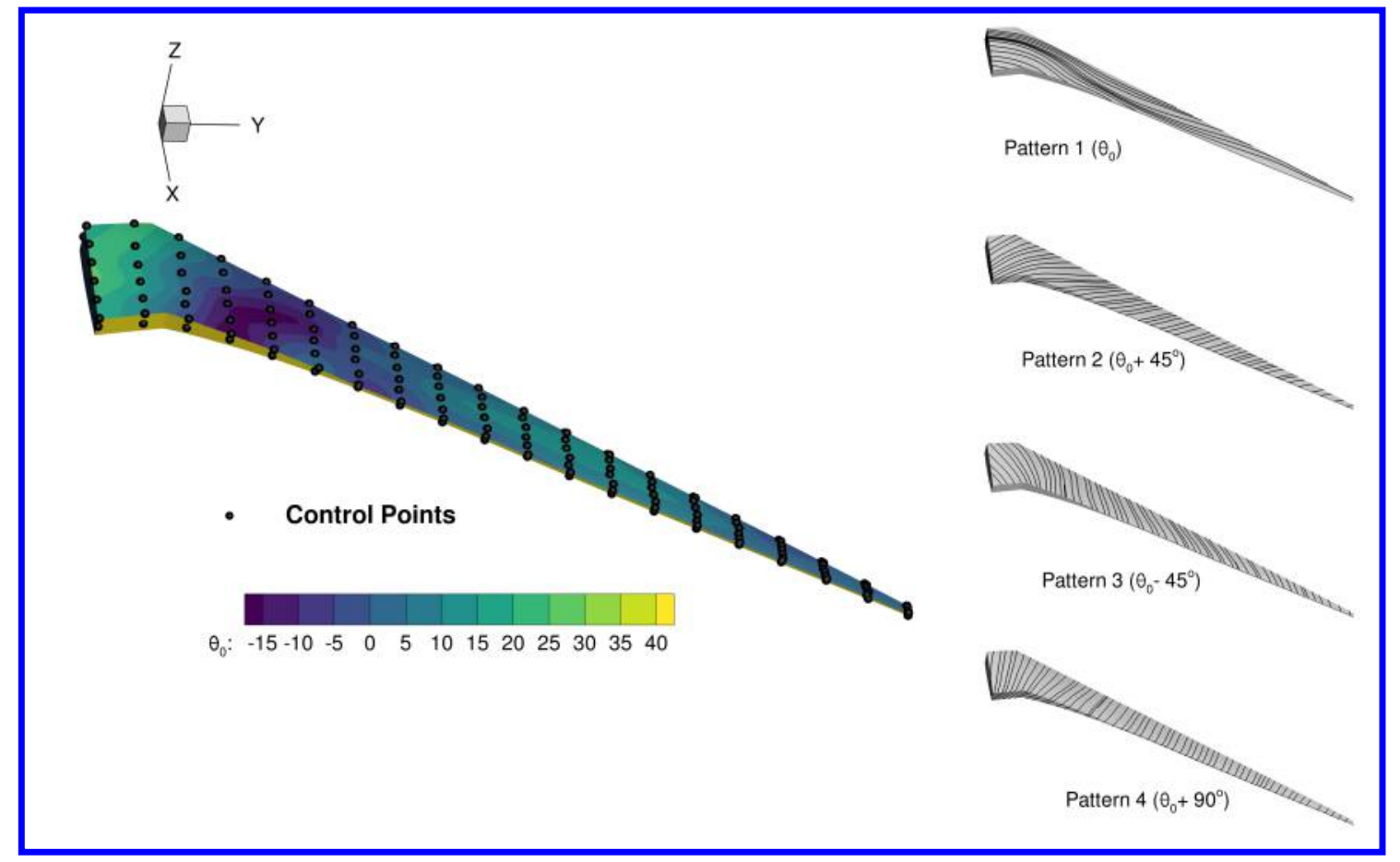

Figure 1. Example of the procedure used to define the main tow path and subsequent patterns

accomplished by defining a unit vector field, $\vec{s}$, as a function of the tow orientation distribution, $\theta(u, v)$, that is at every point tangential to the tow path. The tow curvature can be mathematically defined by taking the curl of the vector field with respect to the parametric variables, $u$ and $v$, and then projecting the resulting vector on the out of plane unit normal, $\vec{n}$, as follows:

$$
\kappa(u, v)=(\nabla \times \vec{s}(\theta)) \cdot \vec{n}
$$

Using the relationship between curvature and turning radius, $r=\frac{1}{\kappa}$, we can compute the tow path radius.

The next constraint considered on the gaps and overlaps of the pre-preg tape. As the tape is laid down to match the streamlines of the ideal vector field, $\vec{s}$, there will be regions in the layup that will be void or where two portions of tape overlap due to fixed width of tape. Gaps and overlaps are desirable, since it takes the design further away from predicted stiffness and mass properties of the ideal model and can potentially lead to unwanted thickness variations. Unfortunately there is no way to exactly predict locations of gaps and overlaps a priori without knowing the tow path of the AFP machine. Regions where gaps and overlap are likely to occur can, however, be qualitatively identified by taking the divergence, $\psi(u, v)$, of the vector field, $\vec{s}$, as follows:

$$
\psi(u, v)=\nabla \cdot \vec{s}(\theta)
$$

By constraining this value it is possible to reduce the number and severity of gap and overlap regions in the manufactured wing.

The final constraint on the tow-steered design is on the layer drop rate of the design. This is how quickly the thickness is allowed to vary across the surface, and can be enforced by constraining the magnitude of the parametric thickness gradient distribution, $\|\nabla t(u, v)\|$. These three constraints are evaluated at the centroid of each element and the aggregated over each panel of the wing box using the Kreisselmeier-Steinhauser (KS) method [17]which can be written as,

$$
K S=c_{\max }+\frac{1}{\rho_{\mathrm{KS}}} \ln \left[\sum_{i=1}^{N} \exp \left(\rho_{\mathrm{KS}}\left(c_{i}-c_{\max }\right)\right)\right]
$$


Where $c_{i}$ is the tow path curvature, divergence, or thickness variation constraint evaluated at the centroid of each element in the aggregation domain. In addition, $c_{\max }$ is the maximum value of all of the $c_{i}$ 's evaluated in the domain. Lastly, $\rho_{\mathrm{KS}}$ is a penalty parameter; the larger this value, the closer the aggregation becomes to the discrete maximum function it is meant to model.

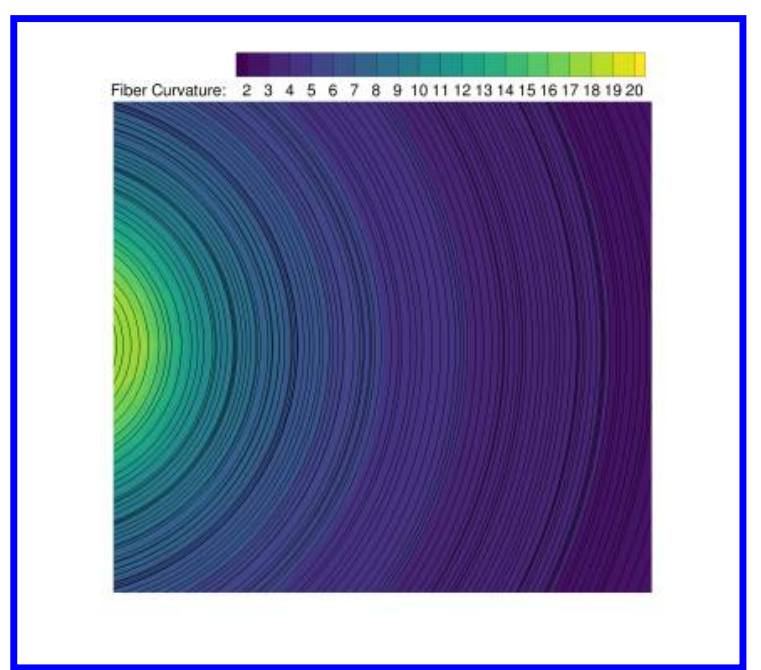

(a) Curvature singularity

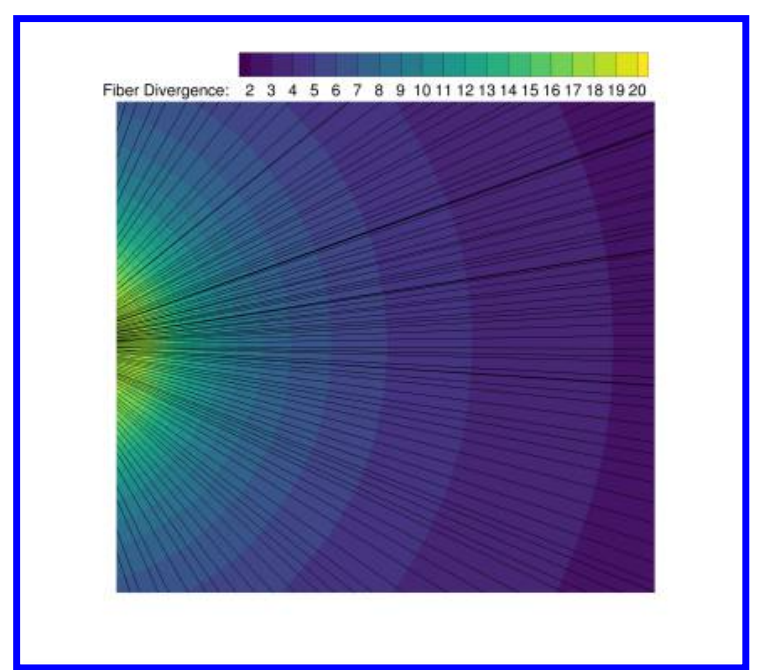

(b) Divergence singularity

Figure 2. Examples of high curvature/divergence vector fields, $\vec{s}(u, v)$

\section{B. Divergence-Curvature Relationship}

Consider a $2 \mathrm{D}$ unit vector field, $\overrightarrow{s_{0}}$ defined by a angle distribution $\theta_{0}(u, v)$ such that:

$$
\overrightarrow{s_{0}}\left(\theta_{0}\right)=\cos \left(\theta_{0}\right) \hat{\mathbf{i}}+\sin \left(\theta_{0}\right) \hat{\mathbf{j}}
$$

For the sake of conciseness we will drop $\theta_{0}$ 's explicit dependence on $u$ and $v$. The streamlines of the vector field $\overrightarrow{s_{0}}$ represent the ideal tow paths of the tow steered laminate ply. If we take the curvature, $\kappa_{0}$, of this vector field as defined in (1) and expand it, we get the relationship:

$$
\kappa_{0}(u, v)=\left(\nabla \times \overrightarrow{s_{0}}\left(\theta_{0}\right)\right) \cdot \vec{n}=\left(\nabla \times\left(\cos \left(\theta_{0}\right) \hat{\mathbf{i}}+\sin \left(\theta_{0}\right) \hat{\mathbf{j}}\right)\right) \cdot \hat{\mathbf{k}}=\frac{\partial \theta_{0}}{\partial u} \cos \left(\theta_{0}\right)+\frac{\partial \theta_{0}}{\partial v} \sin \left(\theta_{0}\right)=\nabla \theta_{0} \cdot \overrightarrow{s_{0}}\left(\theta_{0}\right)
$$

This states that the curvature of vector field is nothing more than the directional derivative of the angle, $\theta_{0}$, in the tangential direction, $\overrightarrow{s_{0}}$, which is a well known identity. Likewise, if we perform the same procedure to compute the divergence given by (2) we get:

$$
\psi_{0}(u, v)=\nabla \cdot \overrightarrow{s_{0}}\left(\theta_{0}\right)=\nabla \cdot\left(\cos \left(\theta_{0}\right) \hat{\mathbf{i}}+\sin \left(\theta_{0}\right) \hat{\mathbf{j}}\right)=\frac{\partial \theta_{0}}{\partial u}\left(-\sin \left(\theta_{0}\right)\right)+\frac{\partial \theta_{0}}{\partial v}\left(\cos \left(\theta_{0}\right)\right)=\nabla \theta_{0} \cdot \overrightarrow{w_{0}}\left(\theta_{0}\right),
$$

where $\overrightarrow{w_{0}}$ is the in plane normal vector to the tangent vector $\overrightarrow{s_{0}}$. Thus, analogously to the curvature, the divergence is the directional derivative of the angle in the transverse direction. This means that in order to reduce the likelihood of gaps and overlaps in the design, the local tow paths should be as close to parallel as possible.

Now let us define a vector field $\overrightarrow{s_{1}}$ parametrized by angle distribution $\theta_{1}$ that is offset from previous vector field $\overrightarrow{s_{0}}$ by a constant angular offset, $\Delta \theta$, such that $\theta_{1}=\theta_{0}+\Delta \theta$. If we now take the curvature of this new vector field, we have:

$$
\kappa_{1}(u, v)=\nabla \theta_{1} \cdot \overrightarrow{s_{1}}\left(\theta_{1}\right)=\nabla \theta_{1} \cdot\left(\cos \left(\theta_{1}\right) \hat{\mathbf{i}}+\sin \left(\theta_{1}\right) \hat{\mathbf{j}}\right)=\frac{\partial \theta_{1}}{\partial u} \cos \left(\theta_{1}\right)+\frac{\partial \theta_{1}}{\partial v} \sin \left(\theta_{1}\right)
$$


Substituting in $\theta_{1}=\theta_{0}+\Delta \theta$ and using the fact that $\frac{\partial \theta_{1}}{\partial u}=\frac{\partial \theta_{0}}{\partial u}$ and $\frac{\partial \theta_{1}}{\partial v}=\frac{\partial \theta_{0}}{\partial v}$, and $\Delta \theta$ is a constant we obtain:

$$
\left.\kappa_{1}(u, v)\right)=\frac{\partial \theta_{0}}{\partial u} \cos \left(\theta_{0}+\Delta \theta\right)+\frac{\partial \theta_{0}}{\partial v} \sin \left(\theta_{0}+\Delta \theta\right)
$$

Finally, using the trigonometric identities $\cos \left(\theta_{0}+\Delta \theta\right)=\cos \left(\theta_{0}\right) \cos (\Delta \theta)-\sin \left(\theta_{0}\right) \sin (\Delta \theta)$ and $\sin \left(\theta_{0}+\Delta \theta\right)=$ $\sin \left(\theta_{0}\right) \cos (\Delta \theta)+\cos \left(\theta_{0}\right) \sin (\Delta \theta)$, collecting like terms, and recognizing the divergence and curvature terms derived previously yields the relationship:

$$
\begin{aligned}
\left.\kappa_{1}(u, v)\right) & =\frac{\partial \theta_{0}}{\partial u}\left(\cos \left(\theta_{0}\right) \cos (\Delta \theta)-\sin \left(\theta_{0}\right) \sin (\Delta \theta)\right)+\frac{\partial \theta_{0}}{\partial v}\left(\sin \left(\theta_{0}\right) \cos (\Delta \theta)+\cos \left(\theta_{0}\right) \sin (\Delta \theta)\right) \\
& =\left(\frac{\partial \theta_{0}}{\partial u} \cos \left(\theta_{0}\right)+\frac{\partial \theta_{0}}{\partial v} \sin \left(\theta_{0}\right)\right) \cos (\Delta \theta)+\left(\frac{\partial \theta_{0}}{\partial u}\left(-\sin \left(\theta_{0}\right)\right)+\frac{\partial \theta_{0}}{\partial v} \cos \left(\theta_{0}\right)\right) \sin (\Delta \theta) \\
& =\kappa_{0} \cos (\Delta \theta)+\psi_{0} \sin (\Delta \theta)
\end{aligned}
$$

In a similar fashion for the divergence, we can derive the relationship:

$$
\psi_{1}(u, v)=-\kappa_{0} \sin (\Delta \theta)+\psi_{0} \cos (\Delta \theta)
$$

These relationships show that the divergence and curvature of two tow steered patterns offset by a constant angle are inherently related. Note that if the offset is exactly $90^{\circ}$ the divergence and curvature swap magnitudes between the two patterns. This effect can be seen most clearly in Figure 2. This means that when considering multiple patterns offset by a constant angle the more stringent of the two constraints (fiber divergence or fiber curvature) ends up being the active constraint for each ply.

\section{Smeared Stiffness Approach}

In order to perform a structural analysis on the tow steered structure the parametric laminate variables, $t(u, v)$ and $\theta_{0}(u, v)$, must first be converted to a parametric stiffness distribution, $\boldsymbol{A}(u, v), \boldsymbol{B}(u, v)$, and $\boldsymbol{D}(u, v)$, using a smeared stiffness approach. First, the homogenized in plane stiffness of the laminate is found based on the ply fraction, $f_{i}$, and the main steered pattern orientation, $\theta_{0}$, as follows:

$$
\overline{\boldsymbol{Q}}(u, v)=\sum_{i=1}^{4} Q\left(\theta_{i}(u, v)\right) \cdot f_{i}
$$

where $\theta_{i}=\left\{\theta_{0}(u, v), \theta_{0}(u, v)+45^{\circ}, \theta_{0}(u, v)-45^{\circ}, \theta_{0}(u, v)+90^{\circ}\right\}, f_{i}=\{0.625,0.125,0.125,0.125\}, \boldsymbol{Q}$ is the stiffness matrix of the ply in the global frame, and $\bar{Q}$ is the homogenized in-plane stiffness matrix of the laminate. Using the parametric thickness distribution, $t(u, v)$, the CLT stiffness matrices can be calculated as follows:

$$
\boldsymbol{A}(u, v)=t(u, v) \cdot \overline{\boldsymbol{Q}}(u, v), \quad \boldsymbol{B}(u, v)=\mathbf{0}, \quad \boldsymbol{D}(u, v)=\frac{t^{3}(u, v)}{12} \cdot \overline{\boldsymbol{Q}}(u, v)
$$

This method neglects the effect order of plies with respect to stiffness and therefore only works well for thick laminates with many repeated plies. The finite-element code then computes the stiffness and mass properties of the models from the nodes using isoparameteric elements based on Gaussian quadrature.

\section{Laminate Failure Analysis}

One of the structural constraints enforced during the optimization is that nowhere in the structure is the laminate allowed to exceed a failure criterion within a safety factor of 1.5. The failure criteria is evaluated at the centroid of each element in the CSM structural model. The failure criterion used for this study was a maximum strain failure criterion, which can be written as follows:

$$
\max \left\{\frac{\epsilon_{1}}{\epsilon_{1_{t}}}, \frac{\epsilon_{1}}{\epsilon_{1_{c}}}, \frac{\epsilon_{2}}{\epsilon_{1_{2}}}, \frac{\epsilon_{2}}{\epsilon_{2_{c}}}, \frac{\gamma_{12}}{\gamma_{12_{s}}},-\frac{\gamma_{12}}{\gamma_{12_{s}}}\right\},
$$


where $\epsilon_{1}, \epsilon_{2}$, and $\gamma_{12}$ are the in-plane strains parallel, transverse, and shearing relative to the layer's local fiber direction, respectively. In addition, $\epsilon_{1_{t}}$ and $\epsilon_{2_{t}}$ are the maximum allowable strain in tension, both parallel and transverse to the fiber direction. Likewise, $\epsilon_{1_{c}}$ and $\epsilon_{2_{c}}$ are the maximum allowable strains in compression. Finally, $\gamma_{12_{s}}$ is the maximum allowable shear strain along the fiber. This constraint is applied as a first ply failure in which no ply can fail. Since the stacking sequence is never explicitly defined in the analysis, we conservatively evaluate failure in the outermost layer for each local fiber orientation given by each unique pattern. After the failure constraint has been evaluated for each element, the constraint is then aggregated over each component of the wing box, (ribs, spars, and wing skin) using the KS aggregation (3).

\section{E. Panel Buckling Analysis}

Each panel of the wing box is also constrained not to buckle for specified load conditions. This constraint is applied in a similar fashion to the failure constraint. A simplified buckling analysis is performed on every panel in the wing box. In this study, stiffeners are not included in the model, which means only buckling of the panel need be analyzed. The panel is assumed to be simply supported along the edges connected to the ribs and due to the high aspect ratio of the panel, the panel is approximated to be infinite in the chordwise direction. Due to the relatively small area of each panel, the stiffness variation in the panel due to thickness and tow orientation changes is assumed to be negligible. To this end, the panel properties are then averaged over the panel to define an equivalent uniform panel. With these assumptions it is then possible to use the well-known analytical formula for the critical buckling load in the longitudinal panel direction, given by,

$$
N_{1_{c r}}=\frac{\pi^{2} \overline{\boldsymbol{D}}_{11}}{L_{x}^{2}},
$$

where $\bar{D}$ is bending stiffness matrix of the equivalent uniform panel, and $L_{x}$ is the panel length in the longitudinal direction. For each element in the panel, a conservative envelope is then enforced such that the stress resultant in each element does not exceed the critical buckling load of the panel. This method has the benefit of being cheap to compute relative to performing a full buckling eigenvalue analysis for each panel, but has the disadvantage of missing some of the tow-steering benefits with respect to buckling. Like the failure constraints, the buckling constraints are evaluated at the centroid of every element and then aggregated over each component group.

\section{Optimization Problem Definition}

We now describe the wing design optimization problem. First, we introduce the high aspect ratio wing model, the uCRM 13.5, that was used in this study. Then, we explain the objective function and its computation procedure will be explained. Finally, we review the optimization design variables and constraints. Two aerostructural optimizations were performed: one with the full tow steering parametrization, which we will call the steered case, and one where the tow orientation was held fixed, the unsteered case, but the laminate thickness and other variables were still allowed to vary. The unsteered case is done in order to isolate the benefits due to the tow steering process. Each optimization requires four aerostructural analysis: a cruise condition for evaluating the performance, and three conditions for which the structural constraints are enforced: a $-1.0 \mathrm{~g}$ dive, $2.5 \mathrm{~g}$ pull-up, and $1.0 \mathrm{~g}$ gust maneuver condition. The parameters for these four conditions are listed in Table 1, where TOGW is the Takeoff Gross Weight of the aircraft. A summary of the tow steered optimization problem is shown in Table 2.

Table 1. Optimization flight condition parameters

\begin{tabular}{lrrrr}
\hline Parameter & Cruise & $2.5 \mathrm{~g}$ & $-1.0 \mathrm{~g}$ & $1.0 \mathrm{~g}$ gust \\
\hline Mach number & 0.85 & 0.64 & 0.64 & 0.86 \\
Altitude (ft.) & 37000 & 0 & 0 & 27300 \\
Weight & LGW $+0.2 \times \mathrm{FB}$ & TOGW & TOGW & TOGW \\
\hline
\end{tabular}




\begin{tabular}{|c|c|c|c|}
\hline & Variable/function & Description & Quantity \\
\hline Minimize & FB & Fuel burn & \\
\hline \multirow[t]{12}{*}{ with respect to } & $\alpha_{i}$ & Angle of attack for each flight case & 4 \\
\hline & $\gamma$ & Wing twist & 10 \\
\hline & $x_{\text {shape }}$ & Wing FFD variables & 216 \\
\hline & $\phi_{i}$ & Tail trim angle for each case & 4 \\
\hline & $\theta_{\text {wing, } \mathrm{U}}^{c p}$ & Wing upper skin tow variables & 120 \\
\hline & $t_{\text {wing, } \mathrm{U}}^{c p}$ & Wing upper skin thickness variables & 120 \\
\hline & $\theta_{\text {wing, } \mathrm{L}}^{c p}$ & Wing lower skin tow variables & 120 \\
\hline & $t_{\text {wing, } \mathrm{L}}^{c p}$ & Wing lower skin thickness variables & 120 \\
\hline & $t_{\text {rib }}$ & Rib thickness variables & 58 \\
\hline & $t_{\mathrm{LS}}^{c p}$ & Leading edge spar thickness variables & 20 \\
\hline & $t_{\mathrm{TS}}^{c p}$ & Trailing edge spar thickness variables & 20 \\
\hline & & Total design variables & 812 \\
\hline \multirow[t]{11}{*}{ subject to } & $L=n_{i} W$ & Load factor & 4 \\
\hline & $c_{m_{y}}^{i}=0$ & Trimmed flight & 4 \\
\hline & $t_{L E} / t_{L E_{\text {init }}} \geq 1.0$ & Leading edge radius & 20 \\
\hline & $t_{T E} / t_{T E_{i n i t}} \geq 1.0$ & Trailing edge thickness & 20 \\
\hline & $\mathrm{KS}_{\text {stress }}<1.0$ & Yield stress & 9 \\
\hline & $\mathrm{KS}_{\text {buckling }}<1.0$ & Buckling & 9 \\
\hline & $t>t_{\mathrm{gage}}$ & Gage thickness & 258 \\
\hline & $-\frac{1}{r_{\min }}<\mathrm{KS}_{\kappa}<\frac{1}{r_{\min }}$ & Fiber path turning radius & 112 \\
\hline & $-\psi_{0}<\mathrm{KS}_{\psi}<\psi_{0}$ & Fiber path divergence & 112 \\
\hline & $\mathrm{KS}_{\|\nabla t\|}<\left\|\nabla t_{0}\right\|$ & Thickness variation & 221 \\
\hline & & Total constraints & 769 \\
\hline
\end{tabular}

Table 2. Tow-steered wing box minimization problem 


\section{A. The uCRM 13.5 Model}

The undeflected Common Research Model 13.5 (uCRM 13.5) is a 13.5 aspect ratio variant of the Common Research Model (CRM) developed by Vassberg et al. [18], which has an aspect ratio of 9. The purpose of the uCRM 13.5, similarly to that of the uCRM 9 developed by Kenway et al. [19], is to define a jig wing geometry and its corresponding structural layout to be used as a baseline for future aerostructural studies. The higher aspect ratio version was developed as a baseline for NASA-funded research into next-generation flexible wings. The high aspect ratio wing planform was defined by taking the planform of the UCRM 9 and increasing the span while keeping the reference area constant until an aspect ratio of 13.5 was achieved, as shown in Figure 3. The outer mold line (OML) was then defined through a single point aerostructural shape optimization of a conventional aluminum wing with the objective of minimizing fuel burn. The specifications for the uCRM 13.5 model are listed in Table 3. A three-view of the uCRM 13.5 is shown in Figure 4. The wing planform and wing box structure used for the optimization are shown in Figure 5. In addition to aerodynamic loads the structural model also considers the inertial effects of the non-structural masses. This includes a 7,500 kg engine along with a number of leading edge and trailing edge masses concentrated at discrete locations along the span of the wing, as shown in Figure 5. The engine is mounted at two locations: on the leading edge spar and a mounting panel located between two rib members. For the aerostructural analysis, the inertial effect of the engine is idealized using point masses connected by rigid body links to the mounting locations. Finally, the analysis also includes the distributed weight of the fuel in the wing during flight. This inertial load is applied as a distributed traction over the lower surface of the wing skin.

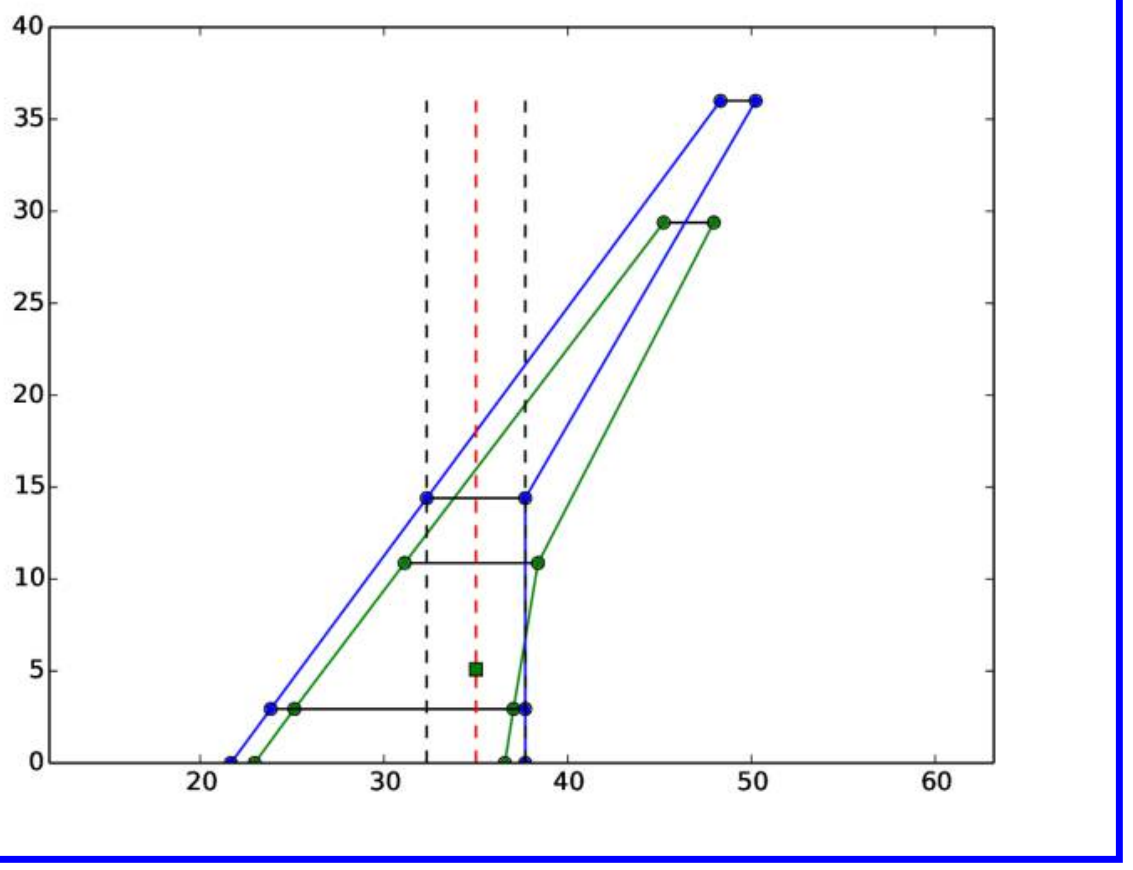

Figure 3. Comparison of uCRM-13.5 wing planform (blue) and CRM-9 planform (green)

\section{B. Objective}

The objective of the optimization problem is to minimize the fuel burn of the aircraft, calculated using the Breguet range equation:

$$
\mathrm{FB}=\mathrm{LGW} \exp \left(\frac{R \mathrm{TSFC}}{V(L / D)}-1\right)
$$




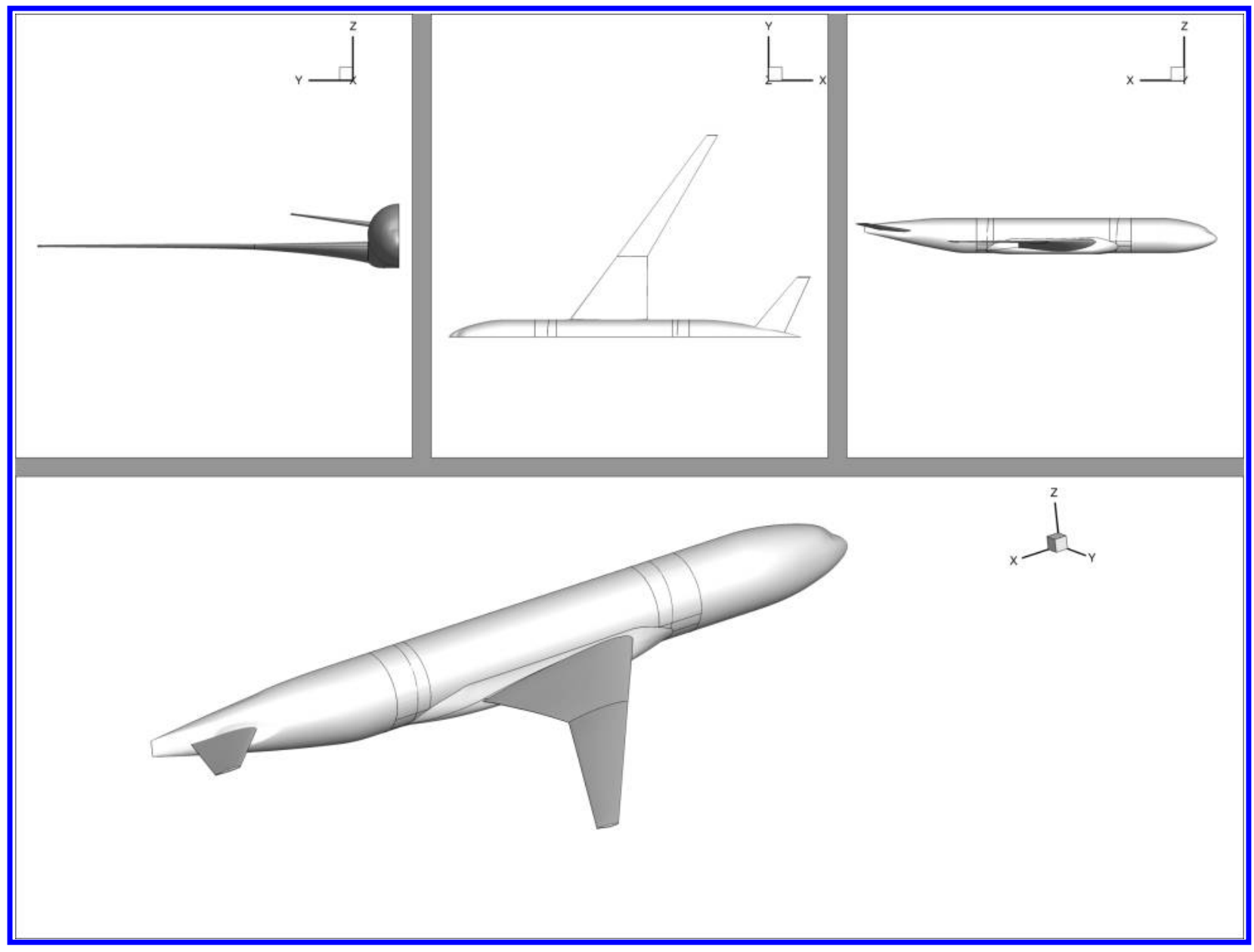

Figure 4. Three-view of the uCRM 13.5 model

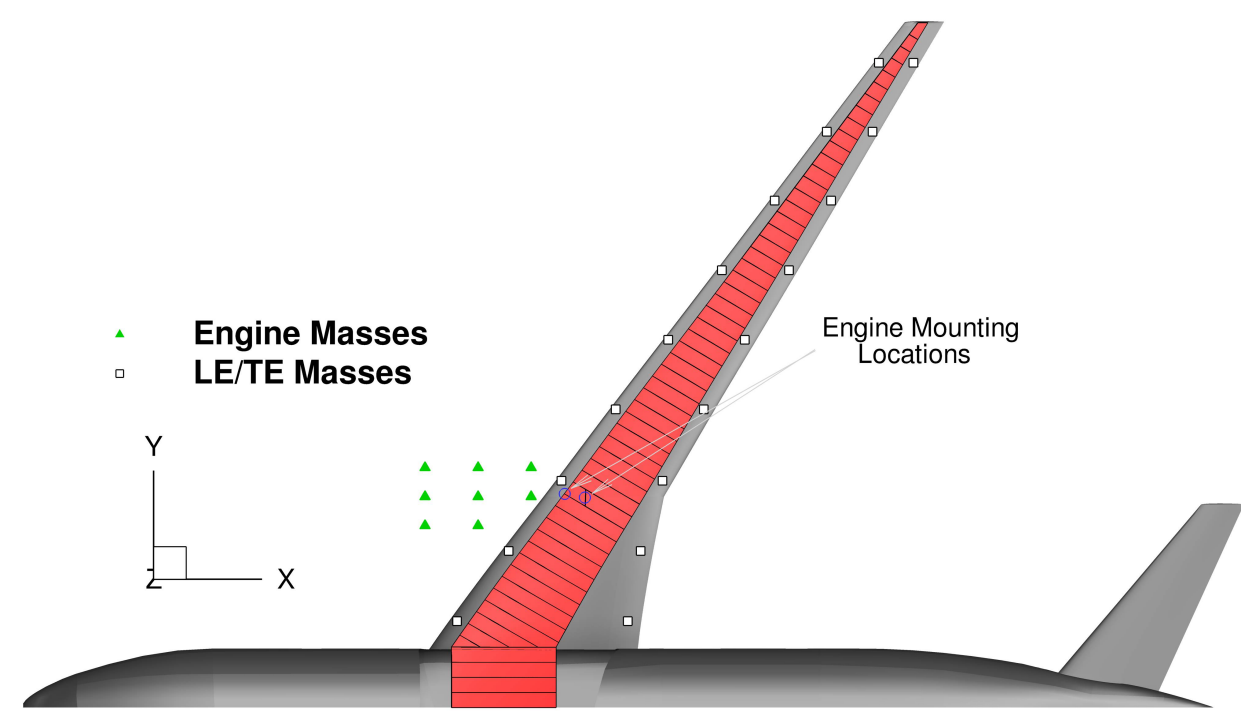

Figure 5. uCRM 13.5 planform and wing box with non-structural masses 
Table 3. uCRM 13.5 specifications

\begin{tabular}{lrl}
\hline Parameter & Value & Units \\
\hline Cruise Mach number & 0.85 & - \\
Cruise lift coefficient & 0.5 & - \\
Initial cruise altitude & 37000 & $\mathrm{ft}$ \\
Span & 72 & $\mathrm{~m}$ \\
Aspect ratio & 13.495 & - \\
Reference wing area & 383.7 & $\mathrm{~m}^{2}$ \\
Sweep (leading edge) & 37.4 & $\circ$ \\
Takeoff gross weight (TOGW) & 297500 & $\mathrm{~kg}$ \\
Maximum landing weight (MLW) & 213180 & $\mathrm{~kg}$ \\
Maximum zero fuel weight (MZFW) & 195040 & $\mathrm{~kg}$ \\
Operational empty weight & 138100 & $\mathrm{~kg}$ \\
Design range & 7725 & $\mathrm{~nm}$ \\
Design payload & 34000 & $\mathrm{~kg}$ \\
Reserve fuel & 15000 & $\mathrm{~kg}$ \\
Initial wing weight & 30286 & $\mathrm{~kg}$ \\
Fixed weight & 107814 & $\mathrm{~kg}$ \\
Thrust specific fuel consumption (TSFC) & 0.53 & $\mathrm{lb} /(\mathrm{lbf} \cdot \mathrm{h})$ \\
\hline
\end{tabular}

where LGW is the landing weight of the aircraft, $R$ is the design range, TSFC is the engine thrust-specific fuel consumption, $V$ is the velocity at cruise, and $L / D$ is the lift-to-drag ratio at cruise. Due to the fact that components such as engine pylons and vertical stabilizer are missing in the aerodynamic model, 35 counts of drag are added to computed drag for every analysis. For simplicity, only the fuel burn during cruise is considered. The landing weight, LGW, is computed using the following formula:

$$
\text { LGW }=1.25 \times W+\text { Fixed Weight }+ \text { Reserve Fuel Weight }+ \text { Secondary Wing Weight }
$$

where $W$ is the weight of the wing box computed from the structural model, and the factor of 1.25 accounts for the weight of missing components in the structural model, such as fasteners and overlaps.

\section{Design Variables}

The design variables used in the optimization can be broken down into three categories: aerodynamic, geometric, and structural. The aerodynamic design variables consists of 4 angles of attacks, one for each flight condition. The geometric design variables consist of 216 wing FFD variables which control the wing cross-sectional shape, 8 twist variables, and 4 horizontal tail incidence angles used to trim each flight condition. The structural design variables consist of 58 rib thickness variables (one for each rib) and 40 spar thickness variables (20 for each spar). The remaining structural variables for the steered case are the tow-steering variables for the upper and lower skins of the wing. These are the thickness and main tow-steering orientation values set at the control points on the upper and lower wing box skins. There are 120 control points for each surface, each of which corresponding to a thickness and tow orientation variable for a total of 480 design variables. For the unsteered optimization, the steer orientation variables are fixed while the thickness variables are free to vary. The total number of design variables for the tow-steered optimization is 812 variables.

\section{Constraints}

In order to produce meaningful results, a number of constraints had to be applied to the optimization, as summarized in Table 2. The structural sizing of the wing box is dictated by three maneuver conditions. The first set of constraints ensure that the lift produced by the aircraft in each condition of flight had to match the weight of the aircraft in that condition multiplied by the load factor of that condition. In this case, there four lift constraints, one for each condition. In addition to this constraint, the pitching moment for each flight condition is constrained to be zero such 
that each flight conditioned is trimmed, for another four constraints. The leading edge radius was constrained from decreasing in order to maintain high lift performance of the wing, and the trailing edge thickness was constrained to ensure manufacturability, adding another 40 constraints. The failure and buckling constraints were aggregated into three groups: ribs and spars, upper skin, and lower skin. These constraints were enforced for the 3 maneuver flight conditions, resulting in a total of 18 constraints: 9 for failure and 9 for buckling.

The material properties for the ribs, spars, and skin are listed in Table 4. The thickness variables for the ribs, spars, and skin are constrained to be no less than $5 \mathrm{~mm}$, adding 258 constraints.

The next set of constraints are related to the divergence and curvature of the tow-steered skins. The fiber curvature was constrained to have a minimum turning radius of $70 \mathrm{in}$, which corresponds to a magnitude of curvature of $0.0143 \mathrm{in}^{-1}$. Due to the relationship between the divergence and curvature of orthogonal plies described in Sec. B, the fiber divergence was constrained to the same value as the curvature. Both constraints were aggregated over each panel of the wing box for a total of 224 constraints (112 on each side). The total number of constraints for the optimization problem adds up to 769 .

Table 4. Mechanical properties for the composites used on skin, ribs, and spars

\begin{tabular}{|c|c|c|c|c|c|c|c|c|c|c|c|c|}
\hline Material & $\begin{array}{r}E_{1} \\
{[\mathrm{GPa}]}\end{array}$ & $\begin{array}{r}E_{2} \\
{[\mathrm{GPa}]}\end{array}$ & $\begin{array}{r}G_{12} \\
{[\mathrm{GPa}]}\end{array}$ & $\begin{array}{r}G_{13} \\
{[\mathrm{GPa}]}\end{array}$ & $\begin{array}{r}G_{23} \\
{[\mathrm{GPa}]}\end{array}$ & $\nu_{12}$ & $\begin{array}{r}X_{t} \\
{[\mathrm{MPa}]}\end{array}$ & $\begin{array}{r}X_{c} \\
{[\mathrm{MPa}]}\end{array}$ & $\begin{array}{r}Y_{t} \\
{[\mathrm{MPa}]}\end{array}$ & $\begin{array}{r}Y_{c} \\
{[\mathrm{MPa}]}\end{array}$ & $\begin{array}{r}S \\
{[\mathrm{MPa}]}\end{array}$ & $\begin{array}{r}\rho \\
{\left[\frac{\mathrm{kg}}{\mathrm{m}^{3}}\right]}\end{array}$ \\
\hline Unidirectional Tape E752LT/AS4 (Skin) & 117.9 & 9.7 & 4.8 & 4.8 & 4.8 & 0.34 & 1648 & 1034 & 64 & 228 & 71 & 1550 \\
\hline PW Fabric AS4/8552 (Ribs and Spars) & 62.1 & 62.1 & 5 & 4.8 & 4.8 & 0.045 & 279 & 266 & 279 & 266 & 70 & 1550 \\
\hline
\end{tabular}

\section{Results}

The results of the tow-steered and unsteered optimization are shown in Figure 6. We can see that there is a $1 \%$ improvement, in the fuel burn of the tow-steered relative to the unsteered wing. From the spanwise lift distribution of the two wings for the cruise and $2.5 \mathrm{~g}$ maneuver, we can see that the difference between the cruise and $2.5 \mathrm{~g}$ maneuver conditions, which corresponds to the passive load alleviation that we seek. Although there is some amount of passive load alleviation, the steered case does not show significantly more load alleviation when compared to the unsteered case. This may be due to the fact that without stiffeners defined in the model, the buckling constraint dominates the optimization. Further evidence for this can be seen in the fact that the thickness distribution changes most noticeably on the lower skin of the wing, where the buckling would be less critical for the design. In addition, a greater difference between optimal cruise and maneuver loads is achieved for other objectives that emphasize structural weight, such as takeoff gross weight [19]. Despite the small difference in load alleviation the optimizer is able to decrease the wing weight of the steered wing by $13 \%$ relative to the unsteered case. This is most probably because the optimizer has the freedom to place the composite tow orientation closer to the orientation of the principal stress in high stressed regions. Figure 7 shows the a comparison between the tow-steered and unsteered patterns for each ply. Due to the buckling constraints and the absence of stringers the wing box panels are much thicker than in a realistic wing box. For both wings, the optimizer adds additional laminate thickness at the engine mounting location to deal with the local stress concentration. The main tow pattern for the upper and lower skin of the wing box both steer back towards the trailing edge at the root and the gradually sweep forward towards the tip. 


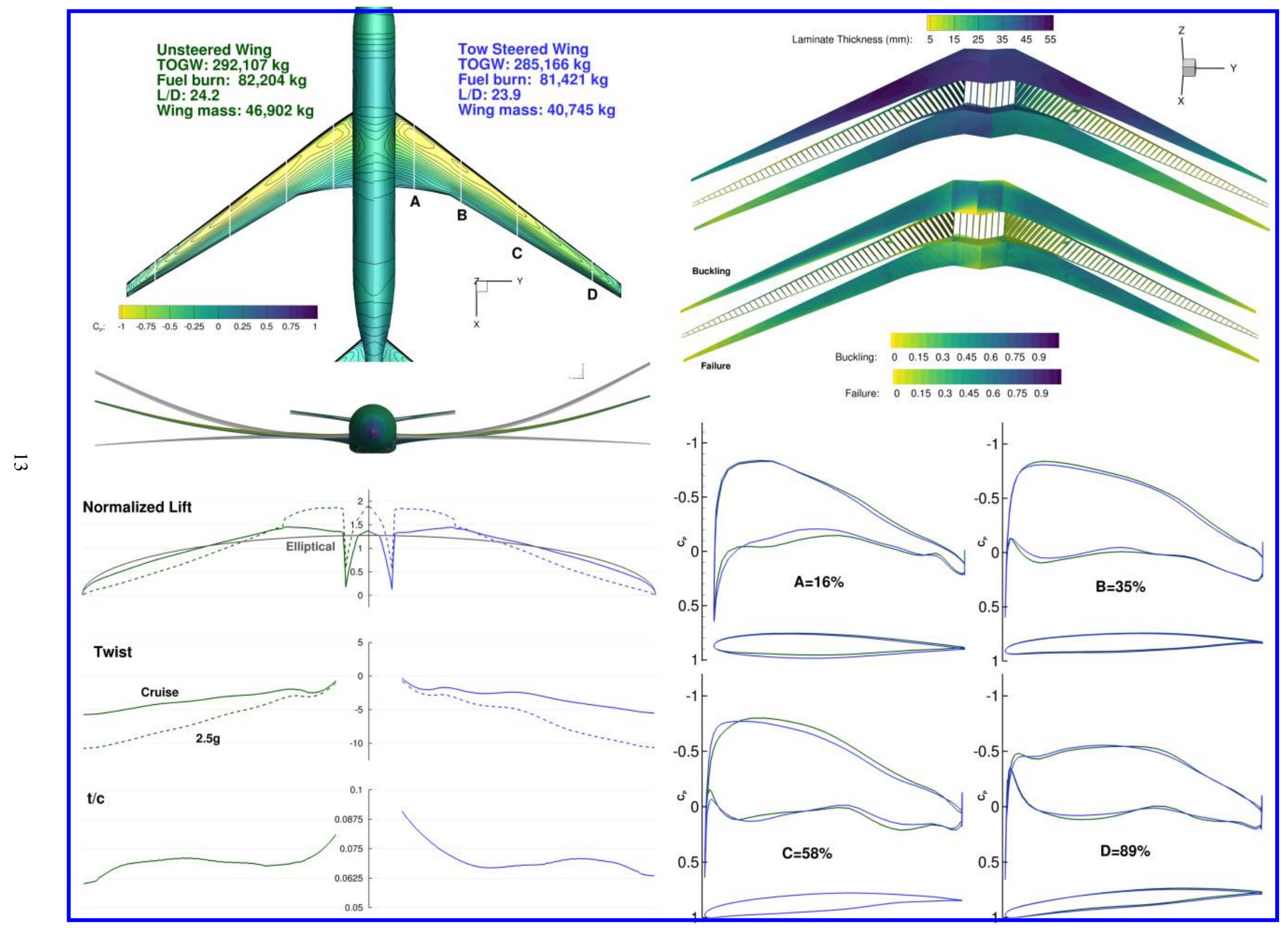

Figure 6. Comparison tow-steered (right) vs unsteered (left) aerostructural optimization results 


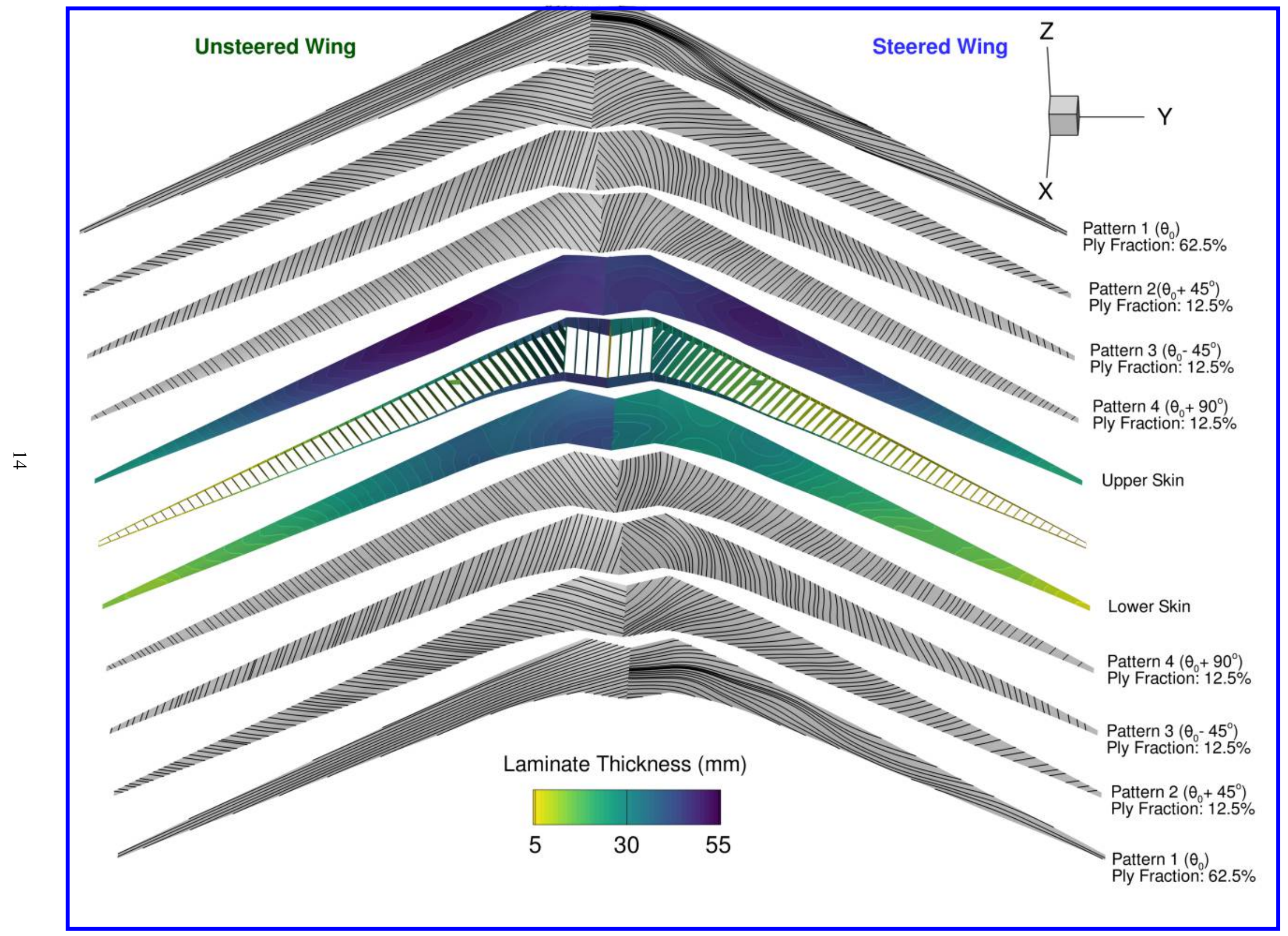

Figure 7. Comparison of optimal tow-steered vs unsteered aerostructural optimized designs 


\section{Conclusion}

We performed a single point aerostructural optimization on both a steered and unsteered wing in order to determine the benefits that tow-steering manufacturing has to offer for the design of flexible high aspect ratio wings. From our optimization results we found that the tow steered wing was able to reduce the structural weight of the wing box by $13 \%$ and the fuel burn by $1 \%$. The differences between the passive load alleviation of the two designs was not as much as was expected. This is likely due to the dominance of the buckling constraints on the optimization due to the lack of stiffeners on the panels. In the future we will add stiffeners to the model in order to remove the undue influence of the buckling constraints, allowing the optimizer to arrive at designs with greater passive load alleviation characteristics. Another study we plan to perform is to evaluate the difference that changing the optimization to a TOGW minimization would have on the design of both the steered and unsteered wing. Finally, adding multiple design conditions to the optimization will allow the optimization to produce tow-steered designs that are more robust. The study presented shows that tow-steered composites have promising potential for reducing structural weight and thereby improving aircraft performance over traditional composites.

\section{Acknowledgments}

The authors would like to acknowledge support from NASA through award No. NNL15AA01C. The authors would also like to acknowledge the following individuals: Dr. Gaetan Kenway from the University of Michigan for his work in the for his work on the definition of the uCRM 13.5 model; Dr. Bret Stanford of NASA Langley Research Center for his help in defining the non-structural mass parameters for the structural model; and Benjamin Smith from the Aurora Flight Sciences company for his insight into the manufacturing process for tow-steered laminates.

\section{References}

-[1] Kenway, G. K. W. and Martins, J. R. R. A., "Multipoint High-Fidelity Aerostructural Optimization of a Transport Aircraft Configuration," Journal of Aircraft, Vol. 51, No. 1, January 2014, pp. 144-160. doi:10.2514/1.C032150.

-[2] Kenway, G. K. W., Kennedy, G. J., and Martins, J. R. R. A., "Scalable Parallel Approach for High-Fidelity Steady-State Aeroelastic Analysis and Derivative Computations," AIAA Journal, Vol. 52, No. 5, May 2014, pp. 935-951. doi:10.2514/1.J052255.

[3] Kennedy, G. J., Kenway, G. K., and Martins, J. R. R. A., “A Comparison of Metallic, Composite and Nanocomposite Optimal Transonic Transport Wings,” Tech. rep., NASA, March 2014, CR-2014-218185.

[4] C. Hvejsel, E. L. and Stolpe, M., "Optimization strategies for discrete multi-material stiffness optimization," Structural and Multidisciplinary Optimization, , No. 44, 2006, pp. 149-163.

- [5] Kennedy, G. J. and Martins, J. R. R. A., "A Laminate Parametrization Technique for Discrete Ply Angle Problems with Manufacturing Constraints," Structural and Multidisciplinary Optimization, Vol. 48, No. 2, August 2013, pp. 379-393. doi:10.1007/s00158-013-0906-9.

-[6] Jutte, C. V., Stanford, B. K., Wieseman, C. D., and Moore, J. B., "Aeroelastic Tailoring of the NASA Common Research Model via Novel Material and Structural Configurations," Proceedings of the AIAA 52nd Aerospace Sciences Meeting, January 2014. doi:doi:10.2514/6.2014-0598.

[7] Stodieck, O., Cooper, J. E., Weaver, P., and Kealy, P., Optimisation of Tow-Steered Composite Wing Laminates for Aeroelastic Tailoring, American Institute of Aeronautics and Astronautics, 2014/05/31 2014. doi:doi:10.2514/6.2014-0343.

- [8] Liem, R., Kenway, G. K. W., and Martins, J. R. R. A., "Multimission Aircraft Fuel Burn Minimization via Multipoint Aerostructural Optimization,” AIAA Journal, Vol. 53, No. 1, January 2015, pp. 104-122. doi:10.2514/1.J052940.

[9] Kenway, G. K., Kennedy, G. J., and Martins, J. R. R. A., “A CAD-Free Approach to High-Fidelity Aerostructural Optimization," Proceedings of the 13th AIAA/ISSMO Multidisciplinary Analysis Optimization Conference, Fort Worth, TX, Sept. 2010, AIAA 2010-9231.

[10] Uyttersprot, L., Inverse Distance Weighting Mesh Deformation, Ph.D. thesis, Delft University of Technology, 2014.

[11] van der Weide, E., Kalitzin, G., Schluter, J., and Alonso, J. J., "Unsteady Turbomachinery Computations Using Massively Parallel Platforms," Proceedings of the 44th AIAA Aerospace Sciences Meeting and Exhibit, Reno, NV, 2006, AIAA 20060421.

-[12] Mader, C. A., Martins, J. R. R. A., Alonso, J. J., and van der Weide, E., “ADjoint: An Approach for the Rapid Development of Discrete Adjoint Solvers,” AIAA Journal, Vol. 46, No. 4, April 2008, pp. 863-873. doi:10.2514/1.29123. 
-[13] Lyu, Z., Kenway, G. K., Paige, C., and Martins, J. R. R. A., “Automatic Differentiation Adjoint of the Reynolds-Averaged Navier-Stokes Equations with a Turbulence Model," 21st AIAA Computational Fluid Dynamics Conference, San Diego, CA, Jul 2013. doi:10.2514/6.2013-2581.

-[14] Kennedy, G. J. and Martins, J. R. R. A., "A Parallel Finite-Element Framework for Large-Scale Gradient-Based Design Optimization of High-Performance Structures," Finite Elements in Analysis and Design, Vol. 87, September 2014, pp. 56-73. doi:10.1016/j.finel.2014.04.011.

-[15] Gill, P., Murray, W., and Saunders, M., "SNOPT: An SQP algorithm for large-scale constraint optimization," SIAM Journal of Optimization, Vol. 12, No. 4, 2002, pp. 979-1006.

-[16] Perez, R. E., Jansen, P. W., and Martins, J. R. R. A., "pyOpt: a Python-Based Object-Oriented Framework for Nonlinear Constrained Optimization," Structural and Multidisciplinary Optimization, Vol. 45, No. 1, January 2012, pp. 101-118. doi:10.1007/s00158-011-0666-3.

-[17] Poon, N. M. K. and Martins, J. R. R. A., "An Adaptive Approach to Constraint Aggregation Using Adjoint Sensitivity Analysis," Structural and Multidisciplinary Optimization, Vol. 34, No. 1, July 2007, pp. 61-73. doi:10.1007/s00158-0060061-7.

[18] Vassberg, J. C., DeHaan, M. A., Rivers, S. M., and Wahls, R. A., "Development of a Common Research Model for Applied CFD Validation Studies," 2008, AIAA 2008-6919.

[19] Kenway, G. K. W., Kennedy, G. J., and Martins, J. R. R. A., "Aerostructural optimization of the Common Research Model configuration," 15th AIAA/ISSMO Multidisciplinary Analysis and Optimization Conference, Atlanta, GA, June 2014, AIAA 2014-3274. 


\section{This article has been cited by:}

1. Timothy R. Brooks, Gaetan K. Kenway, Joaquim Martins. Undeflected Common Research Model (uCRM): An Aerostructural Model for the Study of High Aspect Ratio Transport Aircraft Wings . [Citation] [PDF] [PDF Plus]

2. Ney R. Secco, John Jasa, Gaetan K. Kenway, Joaquim Martins. Component-based Geometry Manipulation for Aerodynamic Shape Optimization with Overset Meshes . [Citation] [PDF] [PDF Plus]

3. Gaetan K. W. Kenway, Joaquim R. R. A. Martins. 2017. Buffet-Onset Constraint Formulation for Aerodynamic Shape Optimization. AIAA Journal 55:6, 1930-1947. [Abstract] [Full Text] [PDF] [PDF Plus]

4. O. Stodieck, J. E. Cooper, P. M. Weaver, P. Kealy. 2017. Aeroelastic Tailoring of a Representative Wing Box Using Tow-Steered Composites. AIAA Journal 55:4, 1425-1439. [Abstract] [Full Text] [PDF] [PDF Plus]

5. Noud P. M. Werter, Jurij Sodja, Roeland De Breuker. 2017. Design and Testing of Aeroelastically Tailored Wings Under Maneuver Loading. AIAA Journal 55:3, 1012-1025. [Abstract] [Full Text] [PDF] [PDF Plus]

6. Gaetan K. Kenway, Asitav Mishra, Ney R. Secco, Karthikeyan Duraisamy, Joaquim Martins. An Efficient Parallel Overset Method for Aerodynamic Shape Optimization . [Citation] [PDF] [PDF Plus]

7. Timothy R. Brooks, Graeme Kennedy, Joaquim Martins. High-fidelity Multipoint Aerostructural Optimization of a High Aspect Ratio Tow-steered Composite Wing . [Citation] [PDF] [PDF Plus]

8. N.P.M. Werter, R. De Breuker. 2016. A novel dynamic aeroelastic framework for aeroelastic tailoring and structural optimisation. Composite Structures . [Crossref] 